Supporting Information for

\title{
Accessible Superchiral Near-Fields Driven by Tailored Electric and Magnetic Resonances in All-Dielectric Nanostructures
}

\author{
Ershad Mohammadi, Ahad Tavakoli, Parisa Dehkhoda, Yasaman Jahani, Kosmas L. \\ Tsakmakidis, Andreas Tittl, \\ Hatice Altug
}

\section{Content:}

S1. Differential absorbed power of a chiral sample for right- and left-circularly polarized light.

S2. Decomposition of optical chirality of hot-spots into the incident and dipolar parts.

S3. Nanophotonic enhancement of the differential transmittance.

S4. The hole-size effect of high refractive index dielectric disks on chiral field formation.

S5. Numerical simulations for additional Pasteur parameter values.

\section{S1. Differential absorbed power of a chiral sample for right- and left-circularly polarized light}

According to Fig. S1, we consider a chiral homogenous sample of arbitrary shape, which is illuminated sequentially with right- and left-circularly polarized lights. The timeharmonic electromagnetic response of such a chiral medium for $\exp (j \omega t)$ convention, can be described by the constitutive relations as:

$$
\begin{aligned}
& \boldsymbol{D}=\varepsilon \boldsymbol{E}-\frac{j \kappa}{c_{0}} \boldsymbol{H} \\
& \boldsymbol{B}=\mu \boldsymbol{H}+\frac{j \kappa}{c_{0}} \boldsymbol{E}
\end{aligned}
$$

where $\varepsilon, \mu$ and $\kappa$ denote the permittivity, permeability and Pasteur parameter of the medium, respectively. The last one accounts for the coupling effects between induced electric and magnetic dipoles and is the origin of the measured chiroptical response of media such as circular dichroism (CD) and optical rotation dispersion (ORD). According to the Poynting's theorem, the time average of power density for such a chiral medium is connected to the optical chirality as":

$$
\operatorname{Re}(\nabla . S)=-\frac{\omega}{2}\left(\operatorname{Im}\{\varepsilon\}|\boldsymbol{E}|^{2}+\operatorname{Im}\{\mu\}|\boldsymbol{H}|^{2}+\frac{4 \operatorname{Im}\{\boldsymbol{\kappa}\}}{k_{0}} C\right)
$$

where $\boldsymbol{S}=\left(\boldsymbol{E} \times \boldsymbol{H}^{*}\right) / 2$ is the complex Poynting's vector and $C$ denotes the optical chirality of local field which is defined as: 


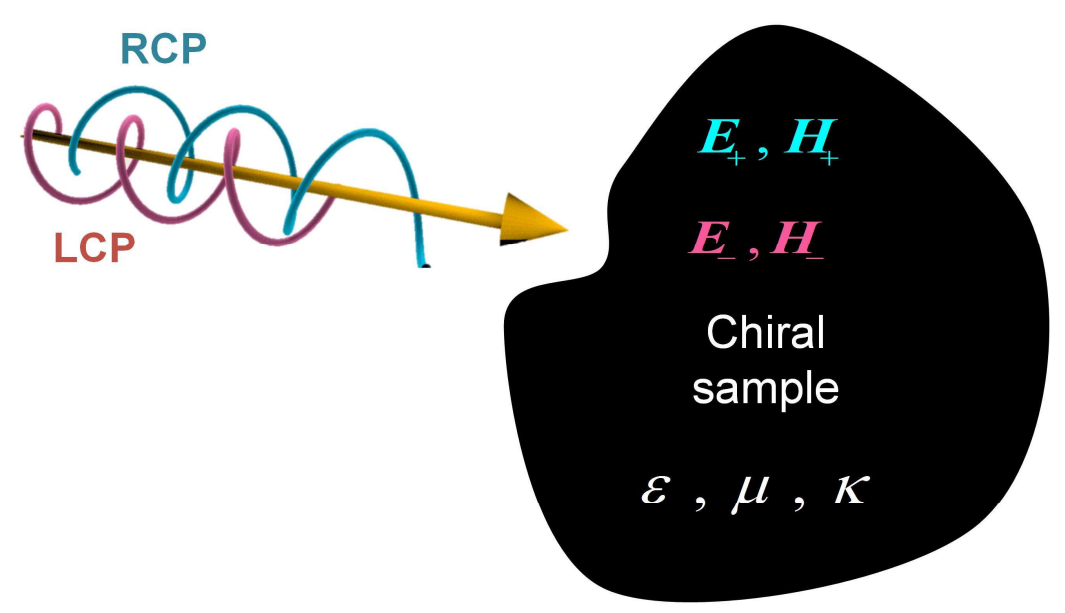

Fig.S1. A homogenous chiral sample of arbitrary shape which is sequentially illuminated with right- and left-handed circularly polarized lights. $\boldsymbol{E}_{+}, \boldsymbol{H}_{+}$and $\boldsymbol{E}_{-}, \boldsymbol{H}_{-}$are the internal local fields corresponding to right- and left-CP illuminations respectively.

$$
C=\frac{-k_{0}}{2 c_{0}} \operatorname{Im}\left\{\boldsymbol{E} . \boldsymbol{H}^{*}\right\}
$$

By integrating the time-averaged power density in Eq. S2 over the entire volume of the chiral sample, the absorbed (dissipated) power for either right- or left-circular illumination, can be written as:

$$
\begin{aligned}
& P_{ \pm}^{a b s}=-\int_{\text {sample }} \operatorname{Re}\left(\nabla \cdot \boldsymbol{S}_{ \pm}\right) \mathrm{dv}= \\
& \frac{\omega}{2} \int_{\text {sample }}\left(\operatorname{Im}\{\varepsilon\}\left|\boldsymbol{E}_{ \pm}\right|^{2}+\operatorname{Im}\{\mu\}\left|\boldsymbol{H}_{ \pm}\right|^{2}+\frac{4 \operatorname{Im}\{\boldsymbol{\kappa}\}}{k_{0}} C_{ \pm}\right) \mathrm{dv}
\end{aligned}
$$

Here "+" and "-." denote the local values inside the chiral sample, upon illumination by right- and left-circular polarization, respectively. It is worth mentioning that these local values may be provided by the near-field of a nanophotonic platform or solely by the circularly polarized illumination. However, in a special case, in which the chiral sample is optically very thin $\left(k_{0} w<<1\right)$, the approximation of $\left|\boldsymbol{E}_{+}\right|=\left|\boldsymbol{E}_{-}\right|,\left|\boldsymbol{H}_{+}\right|=\left|\boldsymbol{H}_{-}\right|$is valid, and the differential absorbed power of the sample with respect to right- and left-circularly polarized light can be obtained as follows: 


$$
\Delta P^{a b s}=P_{+}^{a b s}-P_{-}^{a b s}=-\int_{\text {sample }} \operatorname{Re}\left\{\nabla \cdot\left(\boldsymbol{S}_{+}-\boldsymbol{S}_{-}\right)\right\} \mathrm{dv}=2 c_{0} \operatorname{Im}\{\boldsymbol{\kappa}\} \int_{\text {sample }}\left(C_{+}-C_{-}\right) \mathrm{dv}
$$

\section{S2. Decomposition of optical chirality of hot-spots into the incident and dipolar parts}

Without loss of generality, we consider a plasmonic nanodimer as sketched in Fig. S2, which is composed of two spherical particles placed along the y-axis. The nanodimer is illuminated with a circularly polarized light propagating along the z-axis so that the incident electric and magnetic field components lie in the xy-plane and are expressed as below:

$$
\begin{aligned}
& \boldsymbol{E}_{i n c}=E_{0} \exp \left(-j k_{0} z\right) \hat{e}_{+} \\
& \boldsymbol{H}_{i n c}=j H_{0} \exp \left(-j k_{0} z\right) \hat{e}_{+}
\end{aligned}
$$

where $E_{0}$ and $H_{0}=E_{0} / \eta_{0}$ are the electric and magnetic field amplitudes of the incident light and $\hat{e}_{+}=\hat{x}-j \hat{y}$ is the circular base vector for right-handed polarization.

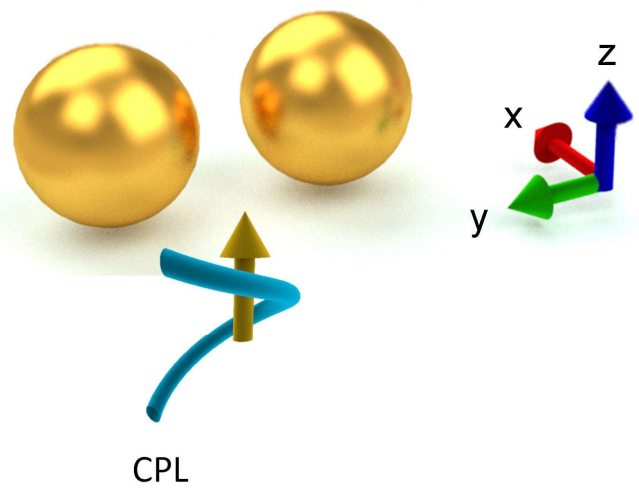

Fig. S2. A nanodimer composed of two spherical particles placed along the $y$-axis and illuminated with a circularly polarized light propagating orthogonal to the dimer axis.

According to Eq. (S3), the optical chirality of incident wave is obtained as:

$$
C\left\{\boldsymbol{E}_{i n c}, \boldsymbol{H}_{i n c}\right\}=\frac{k_{0}}{c_{0}} E_{0} H_{0}
$$

The optical chirality in the gap between the particles can be higher than the value in Eq. (S7). This enhancement can be attributed to the so-called electric and magnetic hot-spots. If we split the electric and magnetic fields of the hot-spots into the incident and dipolar parts as $\boldsymbol{E}_{h}=\boldsymbol{E}_{i n c}+\boldsymbol{E}_{d i p}, \boldsymbol{H}_{h}=\boldsymbol{H}_{i n c}+\boldsymbol{H}_{d i p}$, then the total optical chirality can be decomposed into its different contributing parts as:

$$
C\left\{\boldsymbol{E}_{h}, \boldsymbol{H}_{h}\right\}=\frac{-k_{0}}{2 c_{0}}\left[\operatorname{Im}\left\{\boldsymbol{E}_{i n c} . \boldsymbol{H}_{i n c}^{*}\right\}+\operatorname{Im}\left\{\boldsymbol{E}_{i n c} \cdot \boldsymbol{H}_{d i p}^{*}\right\}+\operatorname{Im}\left\{\boldsymbol{E}_{d i p} . \boldsymbol{H}_{i n c}^{*}\right\}+\operatorname{Im}\left\{\boldsymbol{E}_{d i p} \cdot \boldsymbol{H}_{d i p}^{*}\right\}\right]
$$


In order to obtain the amount of optical chirality enhancement, we normalize the total optical chirality to the optical chirality of incident wave given in Eq. (S7) which leads to:

$$
\frac{C\left\{\boldsymbol{E}_{h}, \boldsymbol{H}_{h}\right\}}{C_{R C P}}=1-\frac{1}{2} \operatorname{Im}\left\{\boldsymbol{E}_{n}^{i n c} \cdot \boldsymbol{H}_{n}^{d i p^{*}}\right\}-\frac{1}{2} \operatorname{Im}\left\{\boldsymbol{E}_{n}^{d i p} \cdot \boldsymbol{H}_{n}^{i n *^{*}}\right\} \frac{1}{2} \operatorname{Im}\left\{\boldsymbol{E}_{n}^{d i p} \cdot \boldsymbol{H}_{n}^{d i p^{*}}\right\}
$$

\section{S3. Nanophotonic enhancement of the differential transmittance}

When a chiral sample is placed in the near field zone of a nanophotonic platform, the super-chiral fields provided by the platform can enhance the differential transmission of the sample with respect to right- and left-circularly polarized light. In order to characterize the amount of this enhancement we first calculate the differential transmission of an individual chiral sample. Fig. S3(a) shows a homogeneous chiral slab of width $w$ and cross-section A which is illuminated sequentially with right- and left-CP lights of equal amplitudes at normal incidence. It can be shown that the input power of either right- or left-handed lights is $P_{ \pm}^{i n c}=E_{0}^{2} A / \eta_{0}$, where $E_{0}$ denotes the electric field amplitude of the incident field and $\eta_{0}$ is the free space wave impedance. Noting that the electromagnetic response of this chiral medium can be described by Eq. (S1), the RCP and LCP waves which pass through the chiral slab experience complex refractive indices of $n_{+}=n+\kappa$ and $n_{-}=n-\kappa$ respectively, where $n=c_{0} \sqrt{\mu \varepsilon}$ is the refractive index of the chiral medium. By neglecting the reflected powers, the transmission coefficients for right and left-circularly polarized lights can be expressed as $^{2}$ :

$$
T_{+}=\exp \left(-2 k_{0} \operatorname{Im}\{n\} w\right) \exp \left(-2 k_{0} \operatorname{Im}\{\kappa\} w\right)
$$

and,

$$
T_{-}=\exp \left(-2 k_{0} \operatorname{Im}\{n\} w\right) \exp \left(2 k_{0} \operatorname{Im}\{\kappa\} w\right)
$$

respectively. Thus, the differential transmittance of the sample is can be calculated as:

$$
\Delta T=T_{+}-T_{-}=-2 \exp \left(-2 k_{0} \operatorname{Im}\{n\} w\right) \sinh \left(2 k_{0} \operatorname{Im}\{\kappa\} w\right)
$$

If we assume that the chiral slab is very thin $\left(k_{0} w<<1\right)$, then the small argument approximation of the expression in Eq. (S12) leads to:

$$
\Delta T=-4 k_{0} \operatorname{Im}\{\kappa\} w
$$

If we assume that the chiral sample has been placed in the near-field zone of an achiral $\left(C_{+}=-C_{-}\right)$nanophotonic platform as shown in Fig. S2(b), according to Eq. (S5) the differential absorbed power of the chiral sample can be expressed as:

$$
\Delta P_{m}^{a b s}=P_{m,+}^{a b s}-P_{m,-}^{a b s}=4 c_{0} \operatorname{Im}\{\kappa\} \int_{\text {sample }} C_{+} \mathrm{dv}
$$



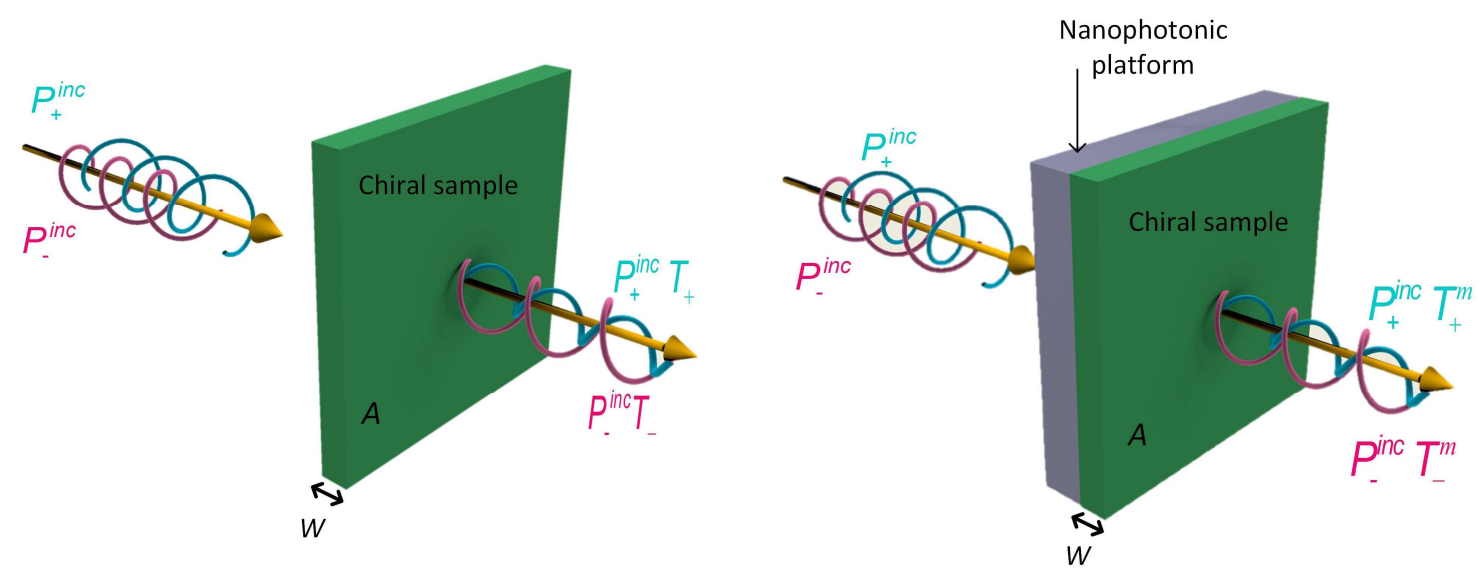

Fig. S3. (a) A chiral sample of width wand cross-section of $A$ which is illuminated with right- and lefthanded circularly polarized lights with input powers of $P_{+}^{\text {inc }}$ and $P_{-}^{\text {inc }}$ respectively. (b) The same chiral sample in (a) which is placed in the near-field zone of a nanophotonic platform and illuminated respectively with right and left circularly polarized lights.

Assuming that the reflected powers are equal for right- and left-handed illuminations, the differential absorbed power $\left(\Delta P_{m}^{a b s}\right)$ is connected to the differential transmitted power $\left(\Delta P_{m}^{t r}=P_{m,+}^{t r}-P_{m,-}^{t r}\right)$ as:

$$
\Delta P_{m}^{t r}=-\Delta P_{m}^{a b s}
$$

On the other side of the sample, the transmitted powers for right and left-handed illuminations are linked to the transmission coefficients as:

$$
\begin{aligned}
& P_{m,+}^{t r}=P_{+}^{i n c} T_{m,+} \\
& P_{m,-}^{t r}=P_{-}^{i n c} T_{m,-}
\end{aligned}
$$

By inserting Eq.(S16) in Eq.(S15) the differential transmittance $\left(\Delta T_{m}=T_{+}^{m}-T_{-}^{m}\right)$ can be connected to the differential absorbed power as:

$$
\Delta T_{m}=\frac{-\Delta P_{m}^{a b s}}{P_{+}^{i n c}}
$$

Next, dividing the nanophotonic enhanced differential transmittance given in Eq. (S17) to the differential transmittance of the individual chiral sample (i.e. without the presence of nanophotonic platform) given in Eq. (S13), provides the magnitude of enhancement as:

$$
\frac{\Delta T_{m}}{\Delta T}=\frac{c_{0} \int_{\text {sample }} C_{+} \mathrm{dv}}{\left(E_{0}^{2} A / \eta_{0}\right)\left(k_{0} w\right)}
$$


If we define the averaged optical chirality as:

$$
C_{+}^{a v}=(1 / V) \int_{\text {sample }} C_{+} \mathrm{dv}(\mathrm{S} 19)
$$

where $V=A w$ is the total volume of the chiral sample, then the expression in Eq. (S18) is reduced to:

$$
\frac{\Delta T_{m}}{\Delta T}=\frac{C_{+}^{a v}}{C_{R C P}}
$$

in which $C_{R C P}$ is the optical chirality of incident right-CP light which was given in Eq. (S7).

\section{S4. The hole-size effect of high refractive index dielectric disks on chiral field formation}

To investigate how the central holes in high refractive index dielectric disks studied in Fig. 4, affect the magnitude of chiral hot-spots, here we consider the Kerker case where the diameter and the height of the dielectric disk are $300 \mathrm{~nm}$ and $130 \mathrm{~nm}$, respectively.

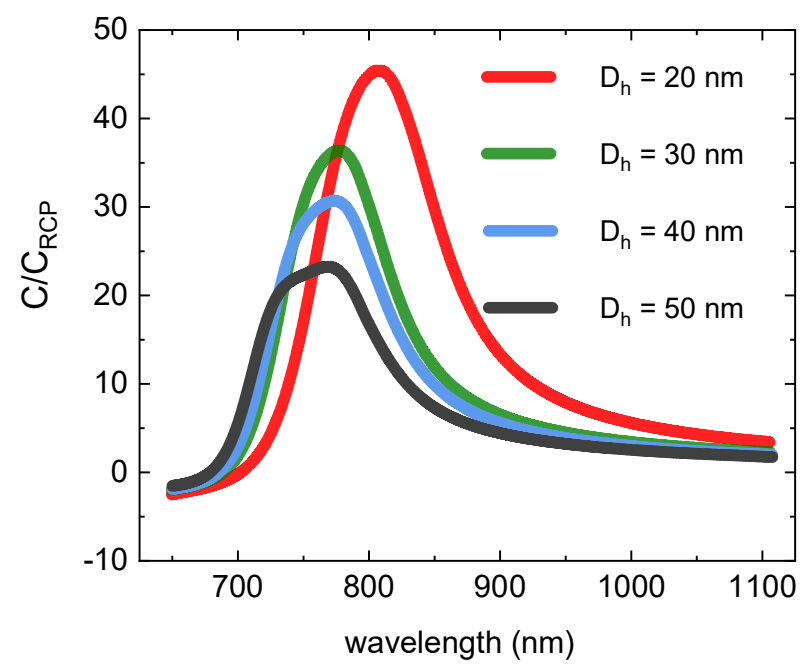

Fig. S4. Investigation of the Kerker disk response for different hole sizes. $D_{h}$ denotes the diameter of the central hole.

In Fig.S4, we have numerically calculated, the optical chirality in the midpoint of the disks with different hole diameters varying from $20 \mathrm{~nm}$ to $50 \mathrm{~nm}$. The results illustrate that by increasing the hole-size, the magnitude of chiral hot-spots is decreased. 


\section{S5. Numerical simulations for additional Pasteur parameter values}

In chiral sensing experiments, small values of the Pasteur parameter below $\kappa=0-0.0001 \mathrm{j}$ can be observed. Fig. S5 presents the simulated differential transmittance spectrum of a holey disk metasurface sensor for a Pasteur parameter with even smaller imaginary part $\left(\kappa=0-0.05 \cdot 10^{-5} \mathrm{j}\right)$, taken from chiral sensing experiments with phenylalanine molecules. ${ }^{3}$

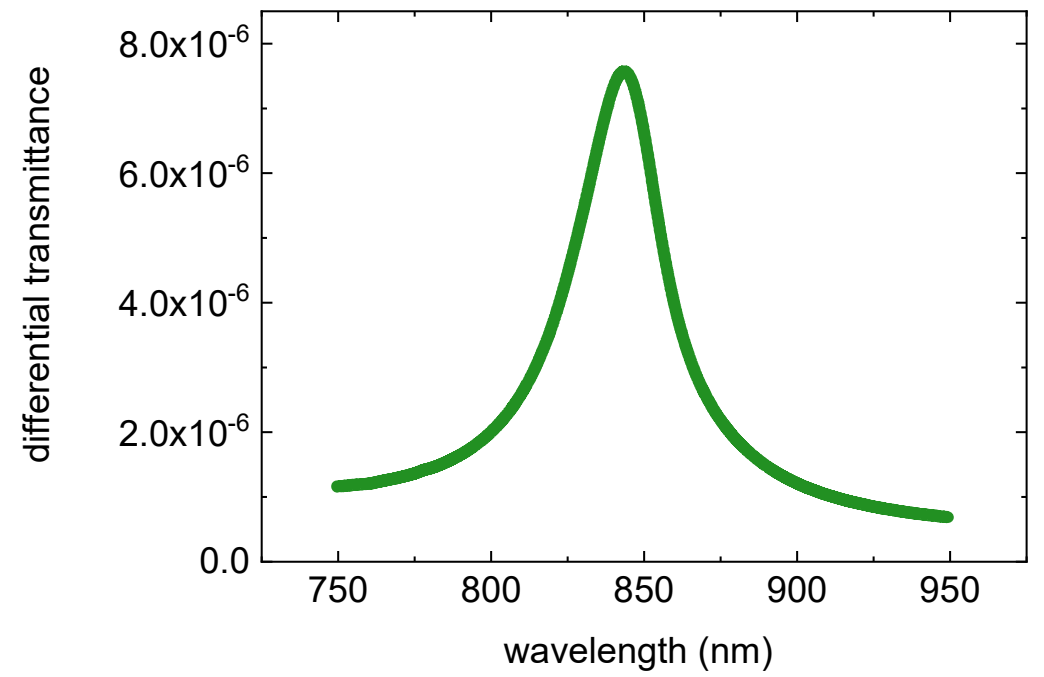

Fig. S5: Differential transmittance spectrum of the holey disk metasurface sensor for a Pasteur parameter value $\kappa=0-0.05 \cdot 10^{-5} \mathrm{j}$ of the adjacent chiral thin film (thickness $50 \mathrm{~nm}$ ).

\section{References}

(1) Mohammadi, E.; Tsakmakidis, K. L.; Askarpour, A. N.; Dehkhoda, P.; Tavakoli, A.; Altug, H. Nanophotonic Platforms for Enhanced Chiral Sensing. ACS Photonics 2018, 5, 2669-2675.

(2) Schäferling, M. Chiral Nanophotonics. Switzerland, Springer Int. Publ. 2017.

(3) García-Guirado, J.; Svedendahl, M.; Puigdollers, J.; Quidant, R. Enantiomer-Selective Molecular Sensing Using Racemic Nanoplasmonic Arrays. Nano Lett. 2018, 18, 6279-6285. 